\title{
The influence of wall roughness on tunnel ventilation friction
}

\author{
Yun-Xiao Xin ${ }^{1, \mathrm{a}, \dagger}$, Ya-Qiong Wang ${ }^{2, \mathrm{~b}}$ and Yi-Kai $\mathrm{Wu}^{3, \mathrm{c}}$ \\ ${ }^{I}$ School of Highway, Chang'an University, 710064, Xi'an, Shaanxi Province, China \\ ${ }^{2}$ Shaanxi Provincial Major Laboratory for Highway Bridge \& Tunnel, Chang'an \\ University, 710064, Xi'an, Shaanxi Province, China \\ ${ }^{3} X i$ 'an Municipal Facilities Management Bureau, Xi'an 710016, China \\ ahapoaaa@126.com, bys08@gl.chd.edu.cn, ${ }^{c} w 51 k @ q q . c o m$
}

\begin{abstract}
To study the effect of surface roughness on the ventilation tunnel wall friction coefficient, the fluent software model was used by changing the height and distance of the tunnel roughness elements and cross-sectional diameter to observe the influence of these control factors in tunnel ventilation. The results showed that: when the air flow velocity is small and brushed the wall, the flow line near the wall substantially parallel to the outer contour of the roughness elements; when the flow rate exceeds a certain value, the flow lines near the wall will have a certain degree of turbulence and reflux. With the same initial wind speed, the more rough wall can make the more friction loss, and the less wall roughness makes the rough elements have less influence on the fluid flow.
\end{abstract}

Keywords: Tunnel ventilation; Friction loss; Numerical simulation; Wall roughness.

\section{Introduction}

The continuous development of China's transportation construction, the number and size of highway tunnels are also increasing [1], the influence of long tunnel has also increased which makes the construction of road tunnel operational after its maintenance. Reasonable ventilation, lighting, design for economic and energy conservation have great significance [2,3]. It is essential to study the process of highway tunnel ventilation in the resistance loss analysis.

Due to road tunnel ventilation research started late, so there are many empirical formulas come from the research of coal mine tunnel ventilation and other industries. However, the road tunnel has its own characteristic, such as ventilation section decrease caused by the passing vehicles. According to the theory of fluid dynamics theory, Wang Xiaowen analyzed the influence of friction loss in tunnel ventilation, got the main affecting factors of the tunnel ventilation resistance [4]. Based on the Zhongnanshan Tunnel measurement data and Nicholas formula, Qiu Yuliang et al suggested the test curve can provide guidance to construction design [5]. The wall roughness of tunnel is one of the important factors affecting the tunnel ventilation resistance, quantitative analysis the value 
of the friction loss in guiding the construction has great significance, but deriving mathematical formulas of the wall roughness on the highway tunnel research is still difficult to achieve [6]. Furthermore, the study on the roughness of highway tunnel wall is still in the theoretical stage, it need a unified standard as its precondition. However, due to the diversity of environmental in tunnel, it is difficult to find the ideal conditions to compare data, so the model experiment and numerical simulation are feasible options to research the friction mechanism $[7,8]$. Using fluent software to create a model for numerical analysis, which can quantitatively compare the friction value with different roughness.

In this context, by the relative roughness of the wall, this paper analyzes the influence caused by height and distance of the tunnel roughness elements and cross-sectional diameter on tunnel ventilation friction, and draw the corresponding wall friction loss and its laws by using the software Fluent analyzes these factors on the fluid flow.

\section{Model}

\subsection{Basic principles.}

Tunnel wall roughness is one of the important factors to affect the value of ventilation friction. In order to quantitatively study the effects of ventilation, the friction loss coefficient is used to measure the value of roughness effect on the tunnel ventilation. Our existing tunnel ventilation specifications provide that the tunnel straight pipe section wall friction loss can be calculated by the following formula. According to the tunnel ventilation standard of China, the friction loss coefficient of tunnel straight section can be calculated by the following formula [9].

$$
\lambda=\frac{1}{\left(1.1138-2 \log \frac{\Delta}{d}\right)^{2}}
$$

Where: $\Delta$ is the average wall roughness, $\mathrm{mm}$, according to Table 1 value; $\mathrm{D}$ is the equivalent diameter of tunnel section, $\mathrm{m}$. 
Table 1. Average wall roughness value

\begin{tabular}{lll}
\hline \multicolumn{2}{c}{ Wall materials and characteristics } & $\Delta / \mathrm{mm}$ \\
\hline \multirow{3}{*}{ Concrete wall } & Smooth & $0.3-0.8$ \\
& Normal & 2.5 \\
& Coarse & $3-9$ \\
\hline \multirow{3}{*}{ Slurry wall } & Smooth & $0.3-0.8$ \\
& Normal & $1.0-2.0$ \\
& Coarse & $2.9-6.4$ \\
\hline Ceramic veneers & - & 1.4 \\
\hline
\end{tabular}

According to hydrodynamic theory, the fluid movement with different flow pattern is applicable to the following formula:

$$
h_{f}=\frac{\lambda \rho L V^{2}}{2 d}
$$

Also:

$$
d=\frac{4 S}{U}
$$

Combining Equations (2) and (3) can be derived:

$$
h_{f}=\frac{\lambda \rho L U V^{2}}{8 S}
$$

Where: $\lambda$ is Darcy coefficient, dimensionless; $\rho$ is the density of fluid, $\mathrm{kg} / \mathrm{m}^{3}$; $L$ is the length of tunnel, $\mathrm{m} ; U$ is the circumference of tunnel section, $\mathrm{m}$; $S$ is the tunnel section area, $\mathrm{m}^{2} ; V$ is the average speed of fluid, $\mathrm{m} / \mathrm{s} ; d$ is the equivalent diameter of tunnel section, $m$.

By setting the air volume inside the tunnel is $Q\left(\mathrm{~m}^{3} / \mathrm{s}\right)$, there is the following relationship:

$$
V=\frac{Q}{S}
$$

Combining Equations (5) and (4) can be derived: 


$$
h_{f}=\alpha \frac{L U}{S^{3}} Q^{2}
$$

Make $R_{f}=\alpha \frac{L U}{S^{3}}, R_{f}$ called friction drag, its unit is: $\mathrm{kg} / \mathrm{m}^{7}$ or $\mathrm{Ns}^{2} / \mathrm{m}^{8}$. Therefore, the Equation (6) can be expressed as:

$$
h_{f}=R_{f} Q^{2}
$$

Tunnel ventilation resistance between the two measuring points can be expressed as:

$$
h_{12}=h^{\prime} C+\frac{\rho_{1}}{2} v_{1}^{2}-\frac{\rho_{2}}{2} v_{2}^{2}
$$

Where: $h_{12}$ is the tunnel friction between the two measuring points, Pa; $h^{\text {'is }}$ the potential difference between the two measuring points, $\mathrm{Pa} ; \rho_{l}, \rho_{2}$ are air densities in two measuring points respectively, $\mathrm{kg} / \mathrm{m}^{3} ; v_{l}, v_{2}$ are average wind speeds at the entry and exit measuring points respectively, $\mathrm{m} / \mathrm{s} ; C$ is the accuracy correction coefficient, dimensionless.

This paper focuses on the impact of the wall roughness on tunnel friction loss, to simplify the analysis, the length of tunnel model is set as $100 \mathrm{~m}$, the potential difference between the two measuring points $h^{\prime}$ can be ignored. According to the practical situation of tunnel ventilation, we can see $h_{f}=h_{12}$, so combining Equation (6) and (8) can get the parameter $\alpha$ :

$$
\alpha=\frac{h_{12} S^{3}}{L U Q^{2}}
$$

\subsection{Calculation parameters.}

In Fluent software, there are two parameters to reflect roughness, Roughness Height $K_{s}$ and Roughness Constant $C_{s}$. Roughness Height $K_{s}$ default value is zero in fluent software, and it means that the tunnel wall is ideal smooth. For uniform particle, $K_{s}$ is the height of particle. For non-uniform particle, $K_{s}$ taking the 
average diameter of particles. For other types of particle, the value of $K_{s}$ should be conversed.

Roughness constant $C_{s}$ is determined by the type of particle, the default value $C_{s}=0.5$ in Fluent software. Combined with $k-\varepsilon$ model, $C_{s}$ can derive the uniform particle roughness. When there is a big difference between particle type and uniform particle, the value of $C_{s}$ can be adjusted to reduce the error. Due to the different types of particles are not selected based on common parameters, so the value of $C_{s}$ is usually experience. In order to obtain more realistic results, it is not necessary to make the mesh size less than $K_{s}$, and it just needs to satisfy the distance between the wall and the grid geometry center more than $K_{s}$.

\subsection{Calculation conditions.}

Due to the tunnel wall is rough, there is always a certain degree of roughness during the construction and operational phase. In order to facilitate analysis, this model set the rough wall surface as a sine curve $t$, as is shown in Fig. 1.By changing the wavelength and amplitude of the curve, this paper simulates the wall roughness of model with different sizes.

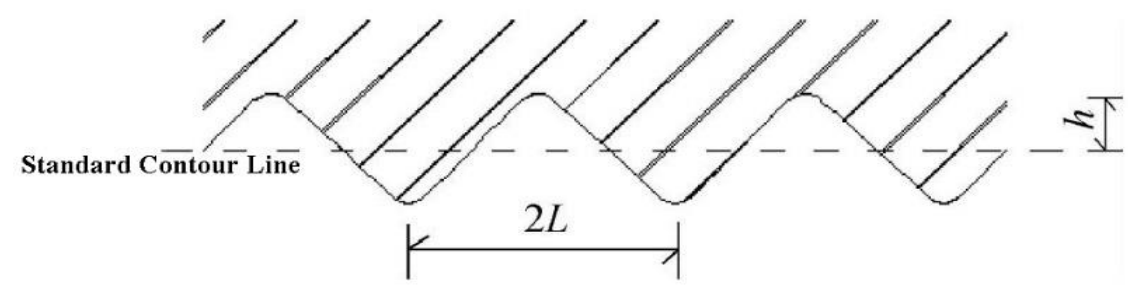

Fig. 1. Sinusoidal type rough wall.

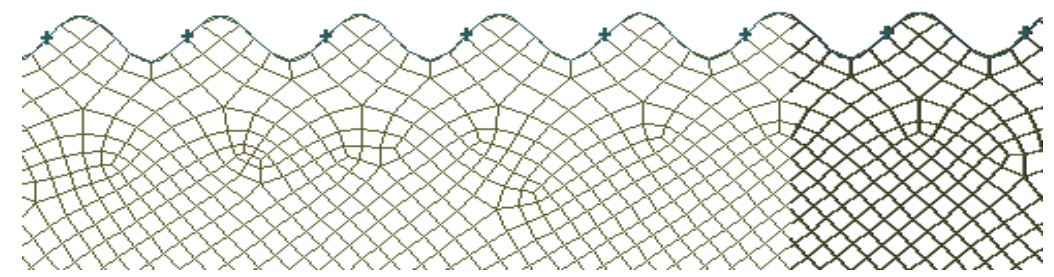

Fig. 2. Grid division of sinusoidal rough model.

To facilitate analysis and comparison of models, the value of air pressure is $101.325 \mathrm{kPa}$, computing environment using the segregated solver with calculate steady-state mode, and the turbulence model is high Reynolds number $k$ - $\varepsilon$ model with SIMPLEC pressure dispersion method. Then selecting $100 \mathrm{~m}$ length model as analyzed segment, and the value of the friction loss is determined by the change of wind speed in this segment. The initial wind speed value is $2 \mathrm{~m} / \mathrm{s}, 4 \mathrm{~m} / \mathrm{s}, 6 \mathrm{~m} / \mathrm{s}$, 
$8 \mathrm{~m} / \mathrm{s}, 10 \mathrm{~m} / \mathrm{s}$ respectively during the calculation process, so that to compare friction changes under different wind speeds.

\section{Calculation of Results and Analysis}

\subsection{The effect of roughnessparticle height.}

To study the influence of particle height on fluid flow, based on the above model, just changing the value of sinusoidal amplitudeh to study with a constant wavelength. So the sinusoid wavelength is set as $2 \mathrm{~L}=0.5 \mathrm{~m}$ in calculation, different values of amplitudeh are $0.01 \mathrm{~m}, 0.03 \mathrm{~m}, 0.05 \mathrm{~m}, 0.07 \mathrm{~m}, 0.1 \mathrm{~m}$. By changing the initial wind speed, friction coefficients can be obtained with different amplitude, as is shown in Fig. 3.

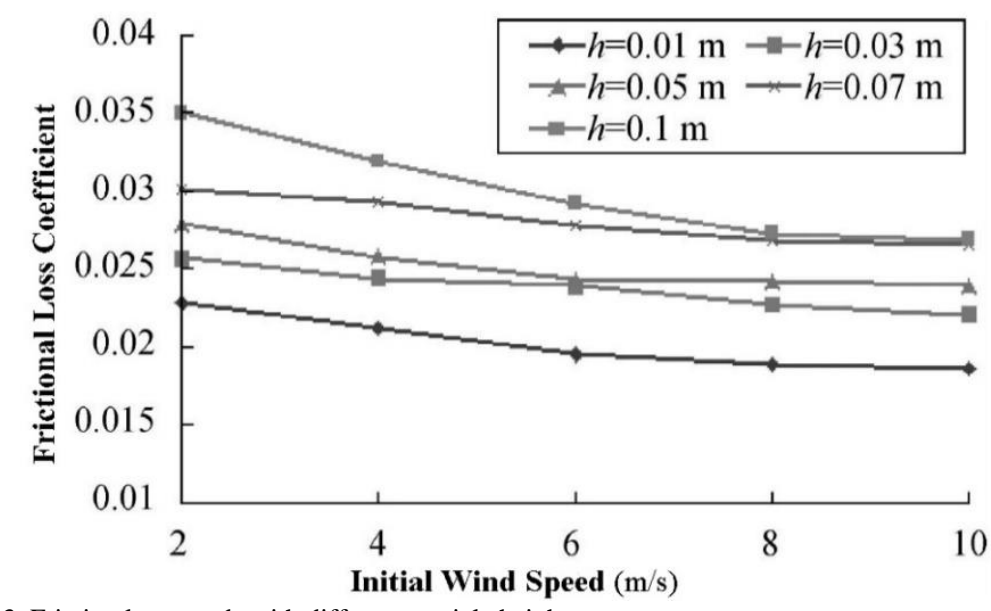

Fig. 3. Friction loss trends with different particle height.

Fig. 3 shows that with the same initial wind speed, the wall roughness height is greater (the roughness is larger), influence of roughness to fluid flow is greater, the corresponding friction loss is greater, and the wind speed difference is larger between 2 test sections. On the contrary, the wall roughness height is smaller (the roughness is smaller), influence of roughness to fluid flow is smaller, it reduce the corresponding friction loss, and the wind speed difference decrease between 2 test sections. For a same sinusoidal model, with the initial wind speed increases, the wind speed in the tunnel also increase and the corresponding friction loss reduce gradually.

In addition, the wind speed of test section 1 sometimes is greater than initial wind speed, because of the location of the test zone. In the numerical simulation with a constant initial wind speed, wind speed in tunnel with airflow direction have a short distance of increased trend and then decrease, but the overall trend is 
reduced. Due to the purpose of the analysis of the different sinusoidal model corresponding to the coefficient of friction loss, it is necessary to test the speed of the two section of the square difference, so it is not related to the initial speed.

\subsection{The effect of roughness particle distance.}

In order to analyze the impact of roughness element distance to fluid flow of sinusoidal model and the change rule of friction loss, the particle heightset as constant value. In the process of numerical calculation, the roughness height of the sine curve model is $\mathrm{h}=0.05 \mathrm{~m}$, and the model of the 6 different rough space is analyzed.

In these 6 models, the length of $100 \mathrm{~m}$ model is selected to analyzed, which is near the end of the tunnel inlet is called the test section 1 , and the other end of the tunnel is called the test section 2. With different initial wind speed $(2 \mathrm{~m} / \mathrm{s}, 4 \mathrm{~m} / \mathrm{s}$, $6 \mathrm{~m} / \mathrm{s}, 8 \mathrm{~m} / \mathrm{s}, 10 \mathrm{~m} / \mathrm{s}$ ) in tunnel inlet, in order to obtain the corresponding wind speed at different initial wind speed between test section 1 and the test section 2 , is obtained. The corresponding friction loss coefficient of the model is obtained by the wind speed of the two test section, as is shown in Fig. 4.

Fig. 4 shows that with the same initial wind speed and roughness height in model, when the element distance increases, the wall tends to be more smooth, and the roughness is relatively reduced, the corresponding friction loss is decreased, the fluid resistance loss caused by roughness wall is reduced, so the wind speed difference between test section 1 and section 2 becomes smaller. On the contrary, when the element distance decreases, the wall tends to be coarser, the corresponding friction loss is increased, the fluid resistance loss caused by roughness wall is increased, so the wind speed difference between test section 1 and section 2 becomes larger. For the same sinusoidal model, with the initial wind speed increases, the wind speed in the tunnel is also relatively increased and friction loss decreased gradually. 


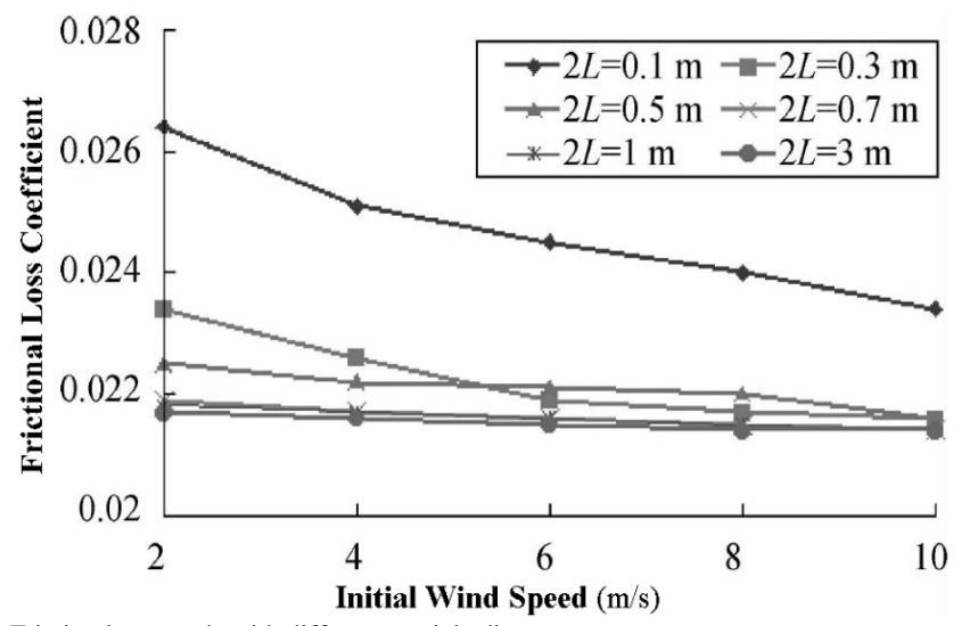

Fig. 4. Friction loss trends with different particle distance.

\subsection{The effect of section diameter.}

To analyze the impact of tunnel section area on friction loss of fluid flow under the same roughness, the rough element distance of sine curve model is set as $2 L=0.5 \mathrm{~m}$, roughness height $h=0.05 \mathrm{~m}$. Then the Gambit software is used to establish5 kinds of two-dimensional models with the same roughness conditions(section shape, element distance and height are same) and different section diameter $\mathrm{D}(4 \mathrm{~m}, 6 \mathrm{~m}, 8 \mathrm{~m}, 10 \mathrm{~m}, 12 \mathrm{~m})$. After the mesh and boundary settings, the mesh files are exported into fluent software with two-dimensional single-precision solver way to analysis. Simulated environment takes the standard atmospheric pressure (101325 $\mathrm{Pa})$, the computing environment using the segregated solver to calculate steady-state mode, turbulence model takes high Reynolds number $k-\varepsilon$ model and use the SIMPLEC pressure dispersion method. The frictional loss coefficient of the corresponding model is obtained by the wind velocity between two test sections, as shown in Fig. 5. 


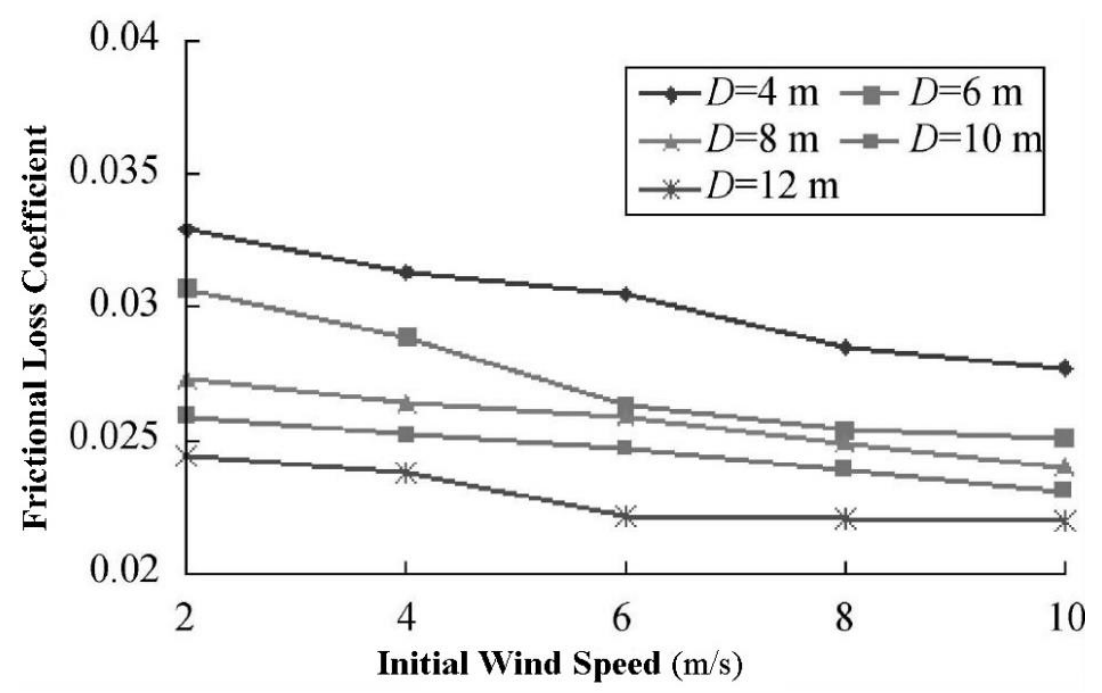

Fig. 5 Friction loss trends with different section diameter.

From Fig. 5, with the same initial wind speed, cross-sectional diameter and roughness degree (the shape, distance and height of roughness elements are constant value), when the wind speed increase, the effect of the wall on fluid flow is relatively reduced, so the wind speed difference between the test sections 1 and section 2 is reduced. On the contrary, with decreasing wind speed, the effect of the wall on fluid flow is relatively increased, so the wind speed difference between the test sections 1 and section 2 becomes larger. For the same initial wind speed, with the cross-sectional diameter increases, the impact of wall on fluid flow is relatively reduced, and friction loss coefficient is reduced accordingly, and the wind speed difference between the test sections 1 and section 2 is reduced. On the contrary, with decreasing cross-sectional diameter, the effect of the wall on fluid flow is relatively increased, so the wind speed difference between the test sections 1 and section 2 becomes larger. For the same sinusoidal model, with the initial wind speed increases, the impact of wall on fluid flow is relatively decreased, and the friction loss is also decreased.

\section{Conclusions}

(1) When the airflow over the wall, and the flow rate is small, flow near the wall parallels the roughness wall contour. When the flow rate exceeds a certain value, the streamline near the wall gradually becomes complex, which is related to the roughness degree and shape of the wall surface, which can generate a certain degree of flow out of the wall surface. 
(2) With a constant initial speed, when the wall roughness increases, the resistance impact of roughness elements is greater on the flow, and the wall friction loss coefficient is larger. On the contrary, when the wall surface roughness degree is smaller, the resistance impact of roughness elements is smaller on the flow, and the wall friction loss coefficient is smaller. However, with the increase of wind speed or section diameter, the influence of wall roughness on fluid flow will be reduced in a certain degree, so that the friction loss coefficient will be reduced accordingly.

(3) When the initial wind speed is constant, the wind speed difference between the two test sections tends to a constant value with the relative decrease of roughness element height or the relative increase of roughness element distance or section diameter. In this condition, it can be considered that the wind power loss tends to the lower limit in this flow field.

\section{Acknowledgment}

This research was financially supported by the Shaanxi Science Foundation of China (2014JM7245) and Central Universities Fundamental Research Innovation Team Project (2013G1502027).

\section{References}

[1]. Hong Kairong. State-of-art and Prospect of Tunnels and Underground Works in China. Tunnel Construction, 2015, 35 (2): 95-107. (in Chinese)

[2]. Xia Fengyong, Xie Yongli, Wang Yaqiong. Complementary Ventilation Modes of Extralong Highway Tunnel.Journal of Traffic and Transportation Engineering, 2014, 14 (6): 27-34. (in Chinese)

[3]. Wang Yaqiong, Xie Yongli, Zhang Sulei. Study on Effect of Nitrogen Oxides onTunnelRequired Ventilation. Journal ofHighway and Transportation Research and Development, 2010, 27 (10): 89-94. (in Chinese)

[4]. Wang Xiaowen. Study on FrictionalLoss for Ventilation of Highway Tunnels. Technology of Highway and Transport, 2004, (3): 87-91. (in Chinese)

[5]. Qiu Yuliang, Li Ningjun, Xie Yongli.Site TestforVentilation Resistance Coefficient ofShotcrete Lining Tunnel. China Journal of Highway and Transport, 2005, 18 (1): 85-88. (in Chinese)

[6]. Zhu Bing, Guan Baoshu, Zheng Daofang. Numerical Simulation of Longitudinal Ventilation of Long Highway Tunnel.Journal of Southwest Jiaotong University, 1999, 34 (2): 8-12. (in Chinese) 
[7]. Tong Yan, Wang Changshun, Chen Liping. Experiment and Numerical Simulation on Natural Ventilation fora Traffic Tunnel with Shafts under Traffic Jam. Chinese Journal of Underground Space and Engineering, 2015, 11 (1): 241-245. (in Chinese)

[8]. Wang Yaqiong, Xie Yongli, Liu Hongzhou. Physical Model Experiment onSemi-transverse Ventilation Air Inlet and Outlet of Sub-sea Tunnel. China Journal of Highway and Transport, 2010, 23 (3): 76-82. (in Chinese) 